Orthopäde $2011 \cdot 40: 125-125$

DOI 10.1007/s00132-010-1707-0

Online publiziert: 26. Januar 2011

(c) Springer-Verlag 2011

C. Klöckner ${ }^{1} \cdot$ U. Weber ${ }^{2}$

${ }^{1}$ Praxis für Wirbelsäulenerkrankungen und Wirbelsäulenchirurgie, Zürich

${ }^{2}$ Klinik für Unfallchirurgie und Orthopädie, DRK-Kliniken Westend, Berlin

\title{
Innovationen in der Wirbelsäulenchirurgie
}

le etabliert haben. Dadurch dass die Anzahl der operativen Eingriffe an der Wirbelsäule nach wie vor zunimmt, ergibt sich natürlich auch die Problematik der ebenso deutlich ansteigenden Anzahl an Revisionseingriffen. Dies ist eine der großen Herausforderungen in der Wirbelsäulenchirurgie. Dabei ist es schon beim Primäreingriff an der Wirbelsäule, insbesondere bei neueren Operationsverfahren notwendig, dass der Chirurg einen Strategieweg für den Fall einer erneuten Problematik im operierten Bereich mit berücksichtigt. Biomechanische Forschungsergebnisse haben uns viele grundlegende Erkenntnisse über die Wirbelsäule gelehrt und sind in den Operationsalltag mit eingeflossen.

Insofern gilt es, die Erkenntnisse und Erfahrungen der mittlerweile praktizierten Operationsverfahren zu sammeln, auch um daraus Schlussfolgerungen für weitere Entwicklungen zu gewinnen.

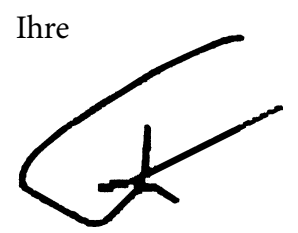

PD Dr. Constantin Klöckner

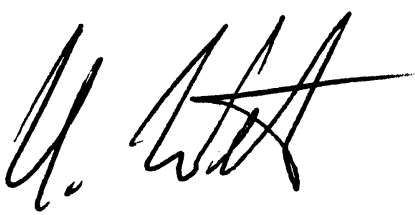

Prof. Dr. Ulrich Weber

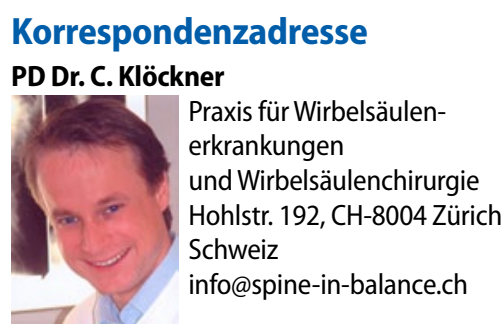

Prof. Dr. U. Weber

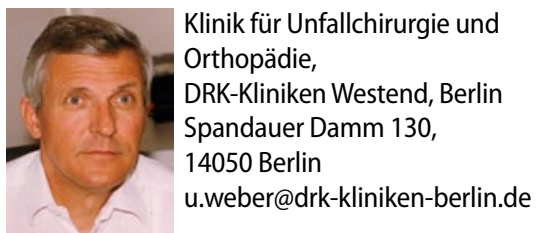

Bestimmte Verfahren wie die endoskopische Nukleotomie haben eine Renaissance erfahren. Andere Zugangswege unter Verwendung zugangsspezifischer Implantate und auch die Fortentwicklung etablierter Zugänge haben vor allem unter dem Aspekt der Operationsmorbidität große Vorteile für den Patienten gebracht. Der Wunsch der bewegungserhaltenden instrumentierten Wirbelsäule ist nach wie vor auf bestimmte Indikationsgruppen beschränkt, die sich mittlerwei- 